\title{
Near $L$-edge Single and Multiple Photoionization of Triply Charged Iron Ions
}

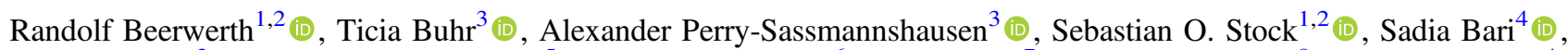 \\ Kristof Holste ${ }^{3}$ (10, A. L. David Kilcoyne ${ }^{5}$ (10), Simon Reinwardt ${ }^{6}$, Sandor Ricz ${ }^{7}$, Daniel Wolf Savin ${ }^{8}$ (1), Kaja Schubert ${ }^{4}$, \\ Michael Martins ${ }^{6}$ (D), Alfred Müller ${ }^{9}$ (D), Stephan Fritzsche ${ }^{1,2}$ (D), and Stefan Schippers ${ }^{3}$ (D) \\ ${ }_{1}^{1}$ Helmholtz-Institut Jena, Fröbelstieg 3, 07743 Jena, Germany; fe3paper@ beerwerth.eu \\ ${ }^{2}$ Theoretisch-Physikalisches Institut, Friedrich-Schiller-Universität Jena, 07743 Jena, Germany \\ ${ }^{3}$ I. Physikalisches Institut, Justus-Liebig-Universität Gießen, Heinrich-Buff-Ring 16, 35392 Giessen, Germany; stefan.schippers@physik.uni-giessen.de \\ ${ }^{4}$ Deutsches Elektronen-Synchrotron DESY, Notkestraße 85, 22607 Hamburg, Germany \\ ${ }_{6}^{5}$ Advanced Light Source, MS 7-100, Lawrence Berkeley National Laboratory, Berkeley, CA 94720, USA \\ ${ }^{6}$ Institut für Experimentalphysik, Universität Hamburg, Luruper Chaussee 149, 22761 Hamburg, Germany \\ ${ }^{7}$ Institute for Nuclear Research, Hungarian Academy of Sciences (MTA Atomki), 4001 Debrecen, Hungary \\ ${ }^{8}$ Columbia Astrophysics Laboratory, Columbia University, 550 West 120th Street, New York, NY 10027, USA \\ ${ }^{9}$ Institut für Atom- und Molekülphysik, Justus-Liebig-Universität Gießen, Leihgesterner Weg 217, 35392 Giessen, Germany \\ Received 2019 August 14; revised 2019 October 20; accepted 2019 October 21; published 2019 December 18
}

\begin{abstract}
Relative cross sections for $m$-fold photoionization $(m=1, \ldots, 5)$ of $\mathrm{Fe}^{3+}$ by single-photon absorption were measured employing the photon-ion merged-beams setup PIPE at the PETRA III synchrotron light source operated at DESY in Hamburg, Germany. The photon energies used spanned the range of $680-950 \mathrm{eV}$, covering both the photoexcitation resonances from the $2 p$ and $2 s$ shells, as well as the direct ionization from both shells. Multiconfiguration Dirac-Hartree-Fock (MCDHF) calculations were performed to simulate the total photoexcitation spectra. Good agreement was found with the experimental results. These computations helped to assign several strong resonance features to specific transitions. We also carried out Hartree-Fock calculations with relativistic extensions taking into account both photoexcitation and photoionization. Furthermore, we performed extensive MCDHF calculations of the Auger cascades that result when an electron is removed from the $2 p$ and $2 s$ shells of $\mathrm{Fe}^{3+}$. Our theoretically predicted charge-state fractions are in good agreement with the experimental results, representing a substantial improvement over previous theoretical calculations. The main reason for the disagreement with the previous calculations is their lack of inclusion of slow Auger decays of several configurations that can only proceed when accompanied by de-excitation of two electrons. In such cases, this additional shake-down transition of a (sub)valence electron is required to gain the necessary energy for the release of the Auger electron.
\end{abstract}

Unified Astronomy Thesaurus concepts: Atomic data benchmarking (2064); Atomic physics (2063); De-excitation rates (2066); Photoionization (2060); Spectral line identification (2073)

Supporting material: machine-readable table

\section{Introduction}

Soft X-ray $L$-shell photoabsorption by $M$-shell iron ions can be important for cosmic objects, ranging from photoionized gas in the vicinity of active galactic nuclei (AGNs) to the near neutral gas of the interstellar medium (ISM). This absorption is largely due to $2 p \rightarrow 3 d$ photoexcitation in $\mathrm{Fe}^{0+}-\mathrm{Fe}^{15+}$, the spectral features of which lie in the $\sim 15-17 \AA$ bandpass ( $\sim 730-830 \mathrm{eV}$; Behar et al. 2001). To help provide reliable iron $L$-shell photoabsorption data for these astrophysical environments, we have carried out a series of combined experimental and theoretical studies. Previously, we presented cross sections for single and multiple photoionization of $\mathrm{Fe}^{+}$ ions in the range of $L$-shell photoexcitation and photoionization (Schippers et al. 2017). Here, we present photoabsorption measurements for $\mathrm{Fe}^{3+}$. Traces of $\mathrm{Fe}^{3+}$ may have been detected in AGN spectra (e.g., Holczer et al. 2005). In the ISM, $\mathrm{Fe}^{3+}$ may also exist in the gas phase (Lee et al. 2009), but equally important, the $\mathrm{Fe}$ in dust grains, when in crystalline structures, may be in the form of $\mathrm{Fe}^{3+}$ (Miedema \& de Groot 2013). Reliable atomic data for gas-phase $\mathrm{Fe}^{3+}$ photoabsorption is needed to distinguish any gas-phase absorption from any solid-matter absorption and for the accurate determination of the iron abundance and its chemical environment. Benchmarking the relevant ionization cross sections by experimental laboratory studies is a prerequisite for such an analysis, as described in more detail by Schippers et al. (2017).

Total photoionization cross sections of $L$-shell electrons for iron have been provided by Reilman \& Manson (1979). Theoretical photoionization cross sections for each subshell are tabulated in the works by Reilman \& Manson (1979), Verner et al. (1993), and Verner \& Yakovlev (1995). Computations of cascade processes that result from inner-shell holes were performed and tabulated by Kaastra \& Mewe (1993), which also includes $L$-shell holes. The body of experimental data on $L$-shell photoionization of iron ions is rather small. Besides our already mentioned previous work on $L$-shell photoionization of $\mathrm{Fe}^{+}$(Schippers et al. 2017), there are two ion-trap based investigations of $\mathrm{Fe}^{+}$ions by Hirsch et al. (2012) and $\mathrm{Fe}^{14+}$ ions by Simon et al. (2010). More recently, Blancard et al. (2018) measured cross sections for photoionization of $\mathrm{Fe}^{q+}$ ions with $6 \leqslant q \leqslant 10$ using the photon-ion merged-beams technique at the synchrotron radiation source SOLEIL focusing on resonant single ionization via the excitation of a $2 p$ electron.

Here, we present our measurements of relative cross sections for up to five-fold ionization of $\mathrm{Fe}^{3+}$ ions via photoexcitation or photoionization of an $L$-shell electron. Our results provide accurate information on the positions and shapes of the 
resonances associated with the excitation of a $2 p$ electron. These data will help to facilitate a reliable identification of $\mathrm{Fe}^{3+}$ photoabsorption features in astrophysical X-ray spectra. Furthermore, we have performed extensive multiconfiguration Dirac-Hartree-Fock (MCDHF) calculations to simulate the experimental spectra and to identify the dominant Auger decay channels. We also carried out Hartree-Fock calculations with relativistic extensions taking into account both photoexcitation and photoionization. Taken together, all these results will be useful for the modeling of the charge balance in astrophysical plasmas.

\section{Experiment}

The experiment was performed at the PIPE end station (Schippers et al. 2014; Müller et al. 2017) of the photon beam line P04 (Viefhaus et al. 2013) at the synchrotron light source PETRA III, which is operated by DESY in Hamburg, Germany. At PIPE, the photon-ion merged-beams technique is used to measure photoionization cross sections of ions. Schippers et al. (2016b) give a recent overview and Schippers et al. (2017) provide a detailed discussion of the experimental method employed here. Typical $\mathrm{Fe}^{3+}$ ion currents in the merged-beam interaction region were $\sim 12 \mathrm{nA}$. The nearly monochromatic photon flux, with an energy spread of $\sim 1 \mathrm{eV}$, was up to $7.8 \times 10^{13} \mathrm{~s}^{-1}$.

Relative cross sections of initial $\mathrm{Fe}^{3+}$ ions for the production of $\mathrm{Fe}^{q+}$ ions $(4 \leqslant q \leqslant 8)$ were measured. As described previously for single and multiple ionization of $\mathrm{Fe}^{+}$ions (Schippers et al. 2017), these measurements are performed individually for each product charge state $q$ by scanning the photon energy from $680 \mathrm{eV}$ up to $950 \mathrm{eV}$. The results are displayed in Figure 1. The measured cross sections span six orders of magnitude. In our previous work on $\mathrm{Fe}^{+}$, we ruled out contributions to the measured signal due to interactions with more than one photon or ionizing collisions off of the residual gas in the apparatus (Schippers et al. 2017). There, it was estimated that such events can be safely disregarded. Because the present data were obtained under very similar experimental conditions, we attribute the measured cross sections in Figure 1 only to processes that involve an initial excitation or ionization of $\mathrm{Fe}^{3+}$ by a single photon.

In principle, the PIPE setup enables measuring photoionization cross sections on an absolute scale. This requires scanning the spatial profiles of the ion beam and the photon beam, from which the geometrical beam overlap factor can be obtained. Unfortunately, such measurements could not be carried out because of a technical problem that could not be solved within the allocated beamtime. Therefore, we multiplied all relative partial cross sections by a common factor such that the crosssection sum

$$
\sigma_{\Sigma}=\sum_{m=1}^{5} \sigma_{m}
$$

matches the theoretical photoionization cross section of Verner et al. (1993) at $692 \mathrm{eV}$ (Figure 2). At these energies, the cross section is dominated by photoionization of the $M$-shell. The rationale for this procedure is that we found excellent agreement between experiment and theory in this energy range in our previous work on photoionization of $\mathrm{Fe}^{+}$where absolute cross sections were measured with a $\pm 15 \%$ total uncertainty at a $90 \%$ confidence limit (Schippers et al. 2017). This suggests that there is a similar uncertainty for the absolute cross-section scale in the present case, after normalization to the theoretical cross section of Verner et al. (1993) as described above. It should be noted that, to a very good approximation, the sum in Equation (1) represents the total photoabsorption cross section, as all the dominant product channels have been measured. The unmeasured $\mathrm{Fe}^{3+}$ product channel, which represents photon scattering, is expected to be insignificant because the fluorescence yield from inner-shell hole states is generally negligible for light elements like iron (McGuire 1972). In this case, our computations confirm the fluorescence yield to be about $1 \%$.

For the determination of the photon-energy scale, the same calibration was used as for our $\mathrm{Fe}^{+}$measurements (Schippers et al. 2017), taking into account the differences in the Doppler shift between the faster $\mathrm{Fe}^{3+}$ ions and the slower $\mathrm{Fe}^{+}$ions. The remaining uncertainty of the experimental photon-energy scale is $\pm 0.2 \mathrm{eV}$.

The ground level of $\mathrm{Fe}^{3+}$ is the $3 d^{5}{ }^{6} S_{5 / 2}$ level. In addition, there are 36 excited $3 d^{5}$ levels that can be populated in the hot plasma of the ECR source. For all of these excited levels, the flight time from the ion source to the photon-ion interaction region is much shorter than the radiative lifetime of the levels (Nahar 2006; Froese Fischer et al. 2008). Consequently, the $\mathrm{Fe}^{3+}$ ion beam consisted of an unknown mixture of groundlevel and excited-level ions. This has to be taken into account when comparing the theoretical calculations with the experimental results, as is discussed in more detail below. Higherexcited, even-parity configurations are expected to play a negligible role as their excitation energies are larger than $15 \mathrm{eV}$. Therefore, their populations are expected to be insignificant for the ion temperatures inferred below for our ion beam.

\section{Theory \\ 3.1. MCDHF Calculations}

To understand and interpret the measured resonance structures, we have performed MCDHF calculations (Grant 2007) to model the photoexcitation cross sections. The background due to direct photoionization was neglected in these models because the fine-structure resolved absolute photoionization cross sections pose major challenges. In addition, independent extensive MCDHF computations were performed to model all the deexcitation pathways due to Auger cascade processes of the $2 s^{-1}$ and $2 p^{-1}$ vacancies created by either photoexcitation or direct photoionization processes. For all MCDHF computations, we utilized the GRASP2K program package (Jönsson et al. 2007, 2013) to generate approximate wave functions, which we describe below. The RATIP code was employed to compute all needed transition rates and relative photoionization cross sections (Fritzsche 2001, 2012).

The computed level structure of the $2 s^{2} 2 p^{6} 3 s^{2} 3 p^{6} 3 d^{5}$ ground configuration of $\mathrm{Fe}^{3+}$ can be seen in the inset of Figure 3. The computed gross structure largely reproduces the experimentally derived energy levels (not shown) reported by Kramida et al. (2018). The most notable observation that can be made here is that the $3 d^{5}{ }^{6} S_{5 / 2}$ ground level is well separated from the more highly excited metastables. However, we have used here the single-configuration approximation without additional corrections for electron correlation effects. As a result, deviations 


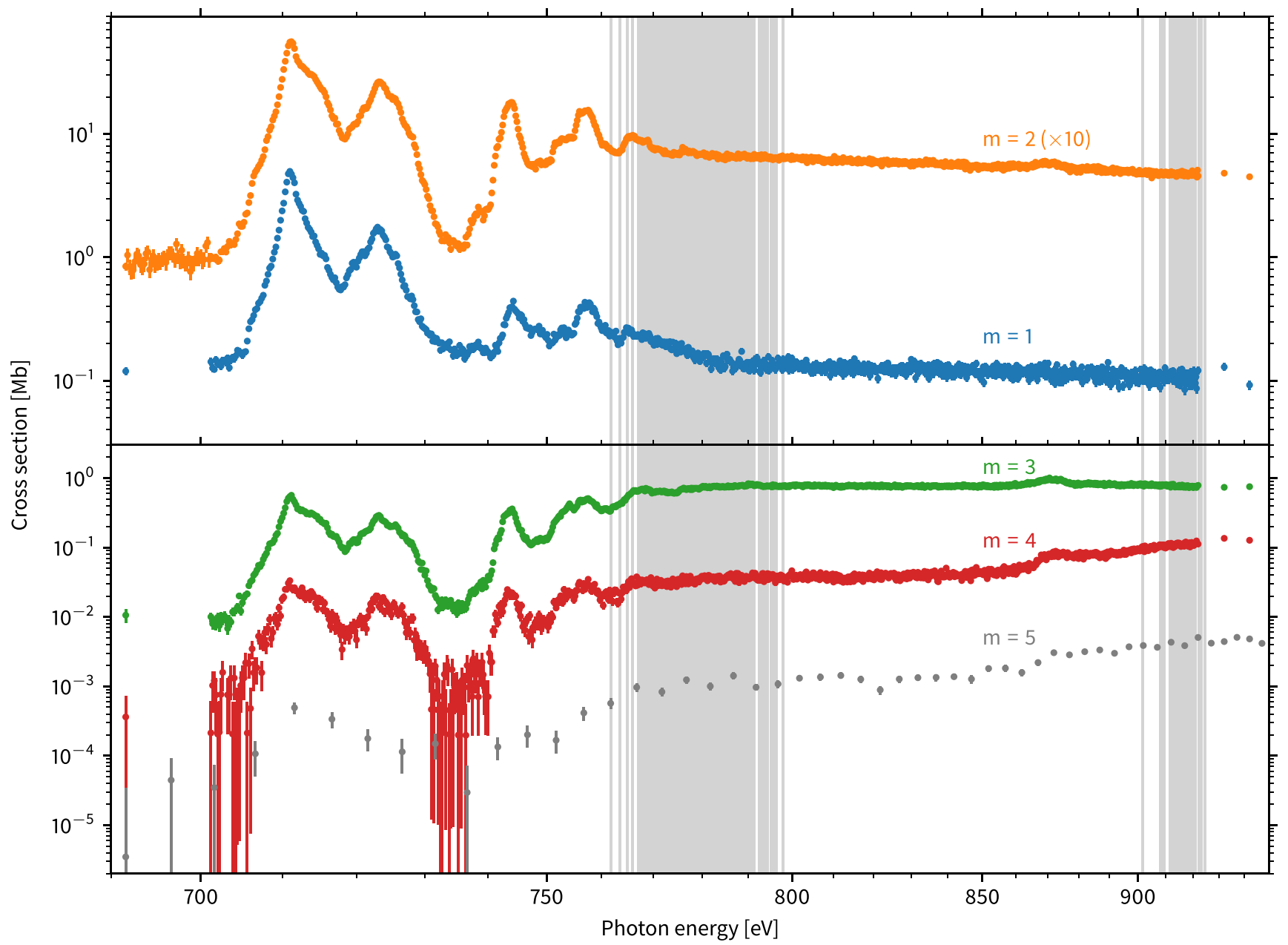

Figure 1. Measured partial cross sections, $\sigma_{m}$, for $m$-fold photoionization of $\mathrm{Fe}^{3+}$. The data are plotted in units of megabarns $(\mathrm{Mb})$, which is $10^{-18} \mathrm{~cm}^{2}$. The partial cross section for $m=2$ was multiplied by a factor 10 to avoid the large overlap with the $m=1$ curve. The vertical gray lines show the computed energy level structure of the $2 s^{2} 2 p^{5} 3 s^{2} 3 p^{6} 3 d^{5}$ and $2 s 2 p^{6} 3 s^{2} 3 p^{6} 3 d^{5}$ configurations, respectively approximately between 760 and $800 \mathrm{eV}$ and between 900 and $925 \mathrm{eV}$. The lowest energy levels correspond to the ionization threshold for $2 p$ and $2 s$ electrons, respectively. For a better view of the low-energy resonance structures, the energy scale has been compressed toward high photon energies according to the formula $E^{\prime}=\log (E-600 \mathrm{eV})$. The absolute cross-section scale was obtained by scaling the summed cross section, given by Equation (1), to the theoretical cross section for photoionization (see the text and Figure 2).

from the measured level energies can be seen. For example, the total energy spread of the ground configuration is computed as $16 \mathrm{eV}$, which is too large by about $2.5 \mathrm{eV}$ (Kramida et al. 2018). Additionally, our computations do not correctly reproduce the level order in some multiplets, due to the limited basis sets used. For example, the first excited ${ }^{4} G$ multiplet has four fine-structure levels ranging from $J=5 / 2$ to $J=11 / 2$, where the latter is lowest in energy and $J=7 / 2$ is highest in energy, separated by about $7.5 \mathrm{meV}$ (Kramida et al. 2018). This order is reversed in our computations, such that $J=5 / 2$ comes out lowest and $J=11 / 2$ highest. As the fine-structure splitting of $0.01 \mathrm{eV}$ is very small compared with the photonenergy spread of $1 \mathrm{eV}$, an incorrect level order within a multiplet does not affect the computed spectra to any significant extent. Furthermore, we note that our singleconfiguration computations reproduce reasonably well the lifetimes calculated by Froese Fischer et al. (2008).

The photoexcitation cross section due to resonant $2 p \rightarrow n d$ photoexcitation was computed based on wave functions for the $3 d^{5}$ ground configuration and the excited $2 s^{2} 2 p^{5} 3 s^{2} 3 p^{6}\left(3 d^{6}+3 d^{5} 4 d+3 d^{5} 5 d\right)$ configurations, taking limited configuration interaction (CI) into account. The contribution of $2 p \rightarrow 4 s$ photoexcitations into the $2 s^{2} 2 p^{5} 3 s^{2} 3 p^{6} 3 d^{5} 4 s$ configuration was found to be negligible and hence has been neglected in the subsequent MCDHF computations. All wave functions were optimized on the basis of the Dirac-Coulomb Hamiltonian, where the energy is obtained as the statistical average over all fine-structure levels in the basis expansion (average-level scheme).

Inner-shell hole states produced by photoexcitation or photoionization will predominantly decay by Auger processes. In the most common two-electron Auger process, one electron fills the inner-shell vacancy and the second electron is released into the continuum producing an ion in the next-higher charge state. A fraction of the Auger decays can result in a so-called shake-up or shake-down transition, where the Auger process is accompanied by an additional excitation or de-excitation, respectively, of a third bound electron, hereafter denoted as a three-electron Auger process. If instead two electrons are simultaneously ejected into the continuum, the process is called direct double-Auger decay. 


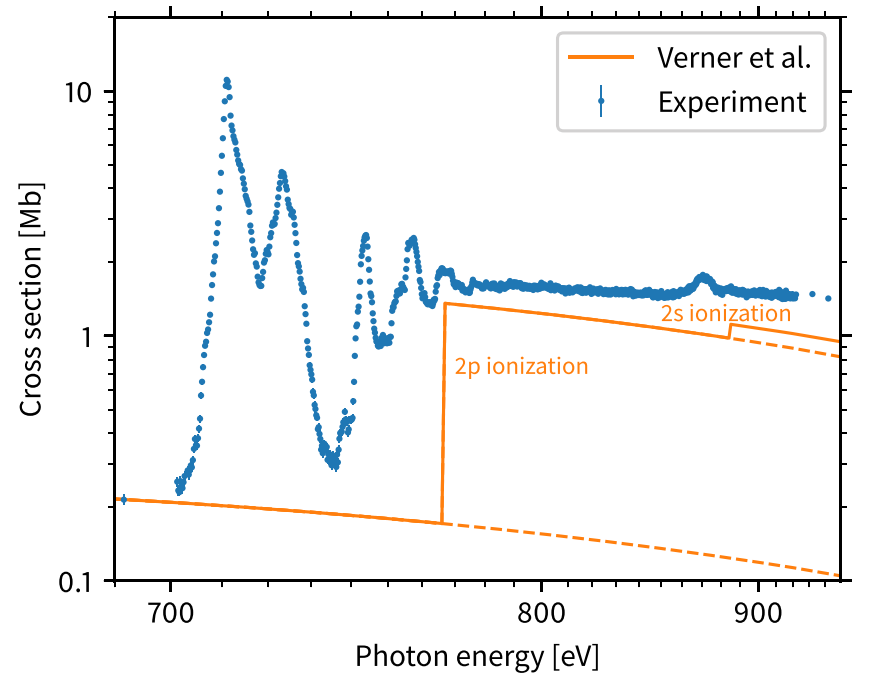

Figure 2. Experimental total photoabsorption cross section given by Equation (1) for $\mathrm{Fe}^{3+}$ (blue circles) and the theoretical cross section for single-photon single ionization of $\mathrm{Fe}^{3+}$ from Verner et al. (1993) (orange line). The steps at 767 and $885 \mathrm{eV}$ correspond to the thresholds for direction photoionization of a $2 p$ and $2 s$ electron, respectively. The dashed lines are the continuation of $M$-shell and $(M+2 p)$-shell photoionization. As in Figure 1, the energy scale is compressed for high energies to enhance the visibility of the low-energy resonance structures.

To model all the de-excitation pathways by sequential Auger decays after, for example, resonant $2 p$ photoexcitation of $\mathrm{Fe}^{3+}$ (forming configuration 2 in Figure 3), we include all electronic configurations that arise from two-electron Auger decay processes emerging from the core-hole excited $2 s^{2} 2 p^{5} 3 s^{2} 3 p^{6} 3 d^{6}$ configuration. All energetically allowed configurations that emerge in this way are shown in Figure 3. We note that more configurations might naively be expected to be accessible but cannot be populated by subsequent Auger emissions due to energy conservation in each step. Therefore, when direct doubleAuger processes, as well as shake-up transitions are neglected, $2 p$ $\rightarrow 3 d$ photoexcited ions can only produce ions up to $\mathrm{Fe}^{6+}$. This limitation is due to energy conservation, as the populated levels with the highest energy in the cascade pathways belong to the $3 s^{-2}$ configuration in $\mathrm{Fe}^{4+}$, labeled 9 in Figure 3. Only photoexcited $2 s$ vacancies lie high enough in energy so that their decay can produce ions in the $\mathrm{Fe}^{7+}$ charge state in this Auger model. The $\mathrm{Fe}^{8+}$ charge state is not significantly populated for any of the photon energies considered here. This is also prevented because $3 s^{-2}$ vacancies in $\mathrm{Fe}^{6+}$ (configuration 21 in Figure 3) are the highest populated configuration after the decay of a $2 p$ vacancy in $\mathrm{Fe}^{5+}$ (configurations 17 and 18 in Figure 3). Even though a decay to $\mathrm{Fe}^{8+}$ is energetically possibly, this fraction is calculated to be around a millionth of a percent and hence orders of magnitude too low to be significant.

Auger cascades resulting from direct photoionization forming $\mathrm{Fe}^{4+}$ are modeled in a very similar manner as for resonantly excited $\mathrm{Fe}^{3+}$. The Auger cascades that emerge from $2 s 2 p^{6} 3 s^{2} 3 p^{6} 3 d^{5}$ and $2 s^{2} 2 p^{5} 3 s^{2} 3 p^{6} 3 d^{5}$ holes are modeled independently. In addition to direct $2 s$ and $2 p$ photoionization, direct photoionization of an $M$-shell electron and subsequent Auger processes have also been considered. As can be seen in Figure 3, all $2 s^{2} 2 p^{6} 3 s 3 p^{6} 3 d^{5}$ holes in $\mathrm{Fe}^{4+}$ (configuration 6 in Figure 3) emit one Auger electron to form $\mathrm{Fe}^{5+}$. However, within the $2 s^{2} 2 p^{6} 3 s^{2} 3 p^{5} 3 d^{5}$ configuration (configuration 5), only the higher-lying levels can undergo an Auger decay to

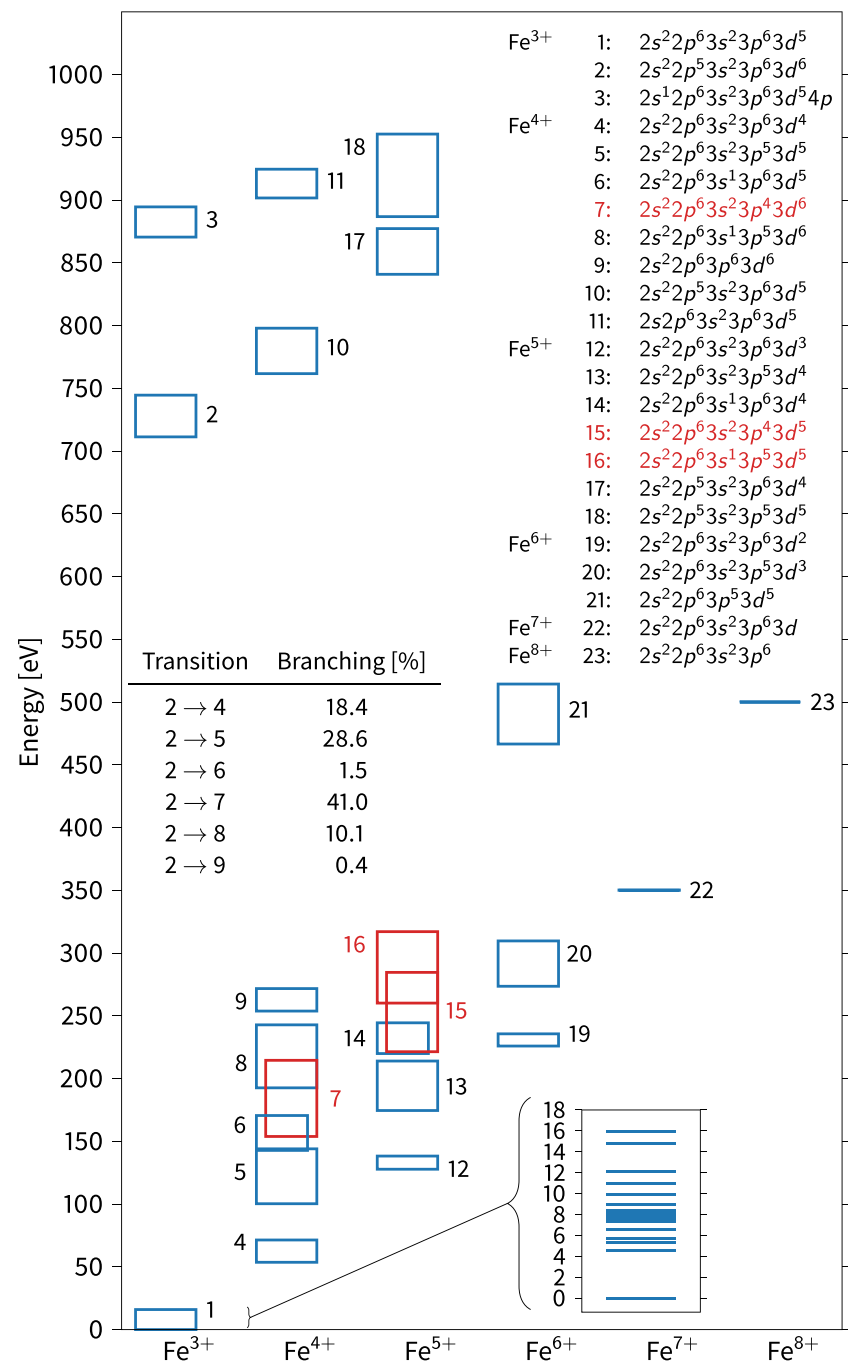

Figure 3. Energy configuration diagram of the hole-state configurations that can be accessed by single-photon excitation or ionization of $\mathrm{Fe}^{3+}$. Some corehole configurations that can be accessed with the current photon energies are listed. All configurations marked in red can, at least partially, decay via threeelectron Auger processes. See Section 4.3 for details. The inset in the lower right corner shows the computed energy levels of the $2 s^{2} 2 p^{6} 3 s^{2} 3 p^{6} 3 d^{5}$ ground configuration. The table in the middle gives the branching ratios of the first Auger decay of the $2 s^{2} 2 p^{5} 3 s^{2} 3 p^{6} 3 d^{6}$ configuration formed by photoexcitation.

$\mathrm{Fe}^{5+}$, while the low-lying levels radiatively relax into the ground configuration of $\mathrm{Fe}^{4+}$.

The total nonradiative decay widths of the 180 fine-structure levels of the $2 s^{2} 2 p^{5} 3 s^{2} 3 p^{6} 3 d^{6}$ configuration vary from 370 to about $550 \mathrm{meV}$. This is expected to be slightly overestimated due to the nonorthogonality of the underlying orbital basis sets for the initial and final wave function expansions. Within the theoretical accuracy, the total nonradiative decay widths of these $2 p$-hole levels created by photoexcitation are similar to the widths of $2 p$ vacancies created by direct photoionization, which also vary from 370 to about $550 \mathrm{meV}$. The $2 s$-hole levels can decay by an Auger process where the $2 s$ hole is filled by a $2 p$ electron, a so-called Coster-Kronig process. This process is much faster than a typical Auger process. Hence, as expected from the Heisenberg uncertainty principle, the associated widths of $3.3-3.7 \mathrm{eV}$ are much larger than those of the $2 p$-hole levels. These widths were only computed for $\mathrm{Fe}^{4+} 2 s$-hole levels resulting from direct $2 s$ ionization. Because the decay widths of $2 p$-hole levels in $\mathrm{Fe}^{3+}$ and $\mathrm{Fe}^{4+}$ are almost 
identical, it is assumed that this also holds for $2 s$ holes. Therefore, we assume that the decay widths of $2 s$ photoexcited $\mathrm{Fe}^{3+}$ levels are within the same range of $2 s$ holes in $\mathrm{Fe}^{4+}$, formed by direct photoionization of a $2 s$ electron in $\mathrm{Fe}^{3+}$.

The cascade model that results from the above considerations gives rise to several thousand fine-structure levels for the intermediate charge states, and hence millions of Auger transitions between those levels. To keep the calculations of the Auger transition rates tractable, it was necessary to constrain the size of the Auger matrices. Therefore, all wave functions were computed in the single-configuration approximation. This approach, detailed in Buth et al. (2018), neglects effects due to CI that become crucial for the description of shake-processes as discussed by Andersson et al. (2015) and Schippers et al. (2016a).

As an additional simplification to make the calculations more readily tractable, one might consider averaging the transition rates between fine-structure levels of the configurations by assuming a statistical population to obtain an average transition rate between configurations as described by Buth et al. (2018). However, such an approach yields results that are very similar to the previous computations by Kaastra \& Mewe (1993), which do not reproduce the experimental findings very well. Therefore, we built the full decay tree between fine-structure levels based on the transition rates computed in the singleconfiguration approximation, while still neglecting radiative losses as they are much slower than Auger processes. Using this approach, we are able to account for the highly nonstatistical population of the fine-structure levels of the initial hole configuration due to the photoexcitation or photoionization of $\mathrm{Fe}^{3+}$.

\subsection{Hartree-Fock Wave Functions with Relativistic Extensions (HFR) Calculations}

Additional calculations have been performed on a CI level utilizing HFR using the Cowan code (Cowan 1981). These calculations account for both photoexcitation and photoionization. CI is included in the initial and the $2 p$ photoexcited or photoionized levels. All possible $L S$-levels are taken into account. The lifetimes, i.e., the line widths of the core-hole resonances, are calculated from the Auger decay rates to various final $\mathrm{Fe}^{4+}$ levels.

For the initial levels the $3 d^{3} 4 s^{2}+3 d^{4} 4 s+3 d^{5}$ configurations are taken into account, with identical $2 s^{2} 2 p^{6} 3 s^{2} 3 p^{6}$ core configurations. Cross sections are calculated for the $2 p$ core excitation from initial level configurations into $2 s^{2} 2 p^{5} 3 s^{2} 3 p^{6} 3 d^{4} 4 s^{2}+$ $2 s^{2} 2 p^{5} 3 s^{2} 3 p^{6} 3 d^{5} 4 s+2 s^{2} 2 p^{5} 3 s^{2} 3 p^{6} 3 d^{6}$ excitations into Rydberg-like $n d(n \geqslant 4)$ orbitals are not taken into account.

As in the MCDHF calculations for the core excited levels, we calculate the Auger transition rates taking into account the decay into the intermediate $\mathrm{Fe}^{4+}$ configurations $3 p^{4} 3 d^{6-k} 4 s^{k} \epsilon(s, d)$ and $3 p^{6} 3 d^{4-k} 4 s^{k} \epsilon(s, d)$ for outgoing $s$ or $d$ waves and $3 p^{5} 3 d^{5-k} 4 s^{k} \epsilon(p, f)$ for $p$ and $f$ waves with $k=0$, 1,2 . Here, the $2 s^{2} 2 p^{6} 3 s^{2}$ core is common for all configurations and $\epsilon$ signifies a free electron. Auger decay channels forming a $3 s^{-2}$ hole are omitted, due to their low transition rates as confirmed by the computed branching ratios shown in the inset table in Figure 3. The calculated lifetime from the Auger transition rates of the core excited levels results in typical line widths in the range of 200-300 meV.

\section{Results and Discussion}

The measured partial cross sections, $\sigma_{m}$, for one- to five-fold ionization of $\mathrm{Fe}^{3+}$ are shown in Figure 1 and are also presented numerically in Table 1 . They span about six orders of magnitude, ranging from almost $10 \mathrm{Mb}$ to less than $0.1 \mathrm{~kb}$. All measured partial cross sections exhibit a complex resonance structure below the $2 p$ ionization threshold. These resonances arise primarily from $2 p \rightarrow n d$ excitations located below and slightly above the $2 p$ ionization threshold. According to our calculation, this threshold is located at $762 \mathrm{eV}$. Verner et al. (1993) obtained a slightly different value of $766.9 \mathrm{eV}$. We expect our result to be more accurate with an expected uncertainty of only a few $\mathrm{eV}$. Due to the presence of metastable species in the $\mathrm{Fe}^{3+}$ ion beam, the threshold can be expected to be somewhat washed out. The 224 fine-structure levels of the $2 s^{2} 2 p^{5} 3 s^{2} 3 p^{6} 3 d^{5}$ configuration of $\mathrm{Fe}^{4+}$ span an energy range of about $35 \mathrm{eV}$ from approximately 762 to $797 \mathrm{eV}$. In Figure 1 these are represented by vertical gray bars.

The calculations show that the measured resonance structures are often blends of many resonance transitions from the ground level, and from the metastable levels of the ground configuration, to the different $2 s^{2} 2 p^{5} 3 s^{2} 3 p^{6} 3 d^{5} n d$ core-hole excited levels. The most prominent feature, which can be discerned in the experimental data, is the $2 p_{3 / 2}-2 p_{1 / 2}$ finestructure splitting of about $15 \mathrm{eV}$ that shows up in the two strong peaks between 700 and $730 \mathrm{eV}$, where the stronger peak at about $711 \mathrm{eV}$ belongs to excitations of $2 p_{3 / 2}$ electrons.

The resonance structure associated with the $2 s \rightarrow n p$ $(n \geqslant 4)$ transitions around $870 \mathrm{eV}$ can be seen in all of the ionization channels. They are much weaker than the features associated with $2 p$ excitations, as the photoabsorption probability is lower due to the fewer number of electrons in the $2 s$ shell. Furthermore, the decay widths of $2 s$ core excited states are about a factor 9 larger than the widths of $2 p$ holes, due to the rapid Coster-Kronig process where the $2 s$ hole is filled by a $2 p$ electron. As a consequence, all $2 s$ resonances have a much larger width and hence appear much weaker compared with the direct ionization background. The $2 s$ ionization threshold is expected at $902 \mathrm{eV}$ according to our calculations and at $885 \mathrm{eV}$ according to the work of Verner et al. (1993). Again, we expect our result to be more accurate, with an uncertainty of only a few eV. This threshold cannot be directly seen in the experimental data. All 74 fine-structure levels of the $2 s 2 p^{6} 3 s^{2} 3 p^{6} 3 d^{5}$ configuration as calculated are shown as gray vertical bars in Figure 1.

The experimental total photoabsorption cross section given by Equation (1) is shown in Figure 2 and compared with the photoionization cross section computed by Verner et al. (1993). The latter includes only direct single-electron photoionization and therefore the resonance features are absent in the computed cross section. At energies above the $2 p$ and $2 s$ resonances the experimental cross section decreases less steeply than the theoretical result. A similar behavior was also observed for $\mathrm{Fe}^{+}$ (Schippers et al. 2017), albeit over a much narrower energy range. Here the deviation between the experimental photoabsorption cross section and the result of Verner et al. (1993) reaches almost a factor of $\sim 1.5$ at the highest experimental photon energy of $950 \mathrm{eV}$. At present, the reason for this discrepancy is not known. One might speculate that the population of metastable levels in the primary ions leads to a change of the photoionization cross section. However, a strong 
Table 1

Measured Partial Cross Sections, $\sigma_{m}$, for $m$-fold Photoionization of $\mathrm{Fe}^{3+}$ ions (Figure 1); Resulting Summed Cross Section, $\sigma_{\Sigma}$, given by Equation (1) (Figure 2); and Mean Product Charge-state, $\bar{q}$, given by Equation (2) (Figure 6(b))

\begin{tabular}{|c|c|c|c|c|c|c|c|}
\hline Energy $(\mathrm{eV})$ & $\sigma_{1}(\mathrm{Mb})$ & $\sigma_{2}(\mathrm{Mb})$ & $\sigma_{3}(\mathrm{Mb})$ & $\sigma_{4}(\mathrm{Mb})$ & $\sigma_{5}(\mathrm{Mb})$ & $\sigma_{\Sigma}(\mathrm{Mb})$ & $\bar{q}$ \\
\hline 691.568 & $0.1191(87)$ & $0.0845(59)$ & $0.0106(24)$ & $0.0004(04)$ & $\ldots$ & $0.214(11)$ & $4.498(30)$ \\
\hline 711.001 & $4.976(53)$ & $5.557(47)$ & $0.542(11)$ & $0.0333(30)$ & $\ldots$ & $11.109(71)$ & $4.6069(36)$ \\
\hline 711.602 & $4.021(34)$ & $4.938(34)$ & $0.4561(85)$ & $0.0264(19)$ & $0.000492(94)$ & $9.441(48)$ & $4.6280(29)$ \\
\hline 723.021 & $1.756(31)$ & $2.609(32)$ & $0.2852(81)$ & $0.0198(23)$ & $\ldots$ & $4.670(45)$ & $4.6935(57)$ \\
\hline 731.636 & $0.2067(80)$ & $0.1873(66)$ & $0.0204(18)$ & $0.00195(53)$ & $0.000149(59)$ & $0.416(11)$ & $4.562(15)$ \\
\hline 744.057 & $0.397(16)$ & $1.803(27)$ & $0.3603(89)$ & $0.0222(24)$ & $\ldots$ & $2.582(33)$ & $5.0030(71)$ \\
\hline 801.754 & $0.1333(64)$ & $0.637(12)$ & $0.789(11)$ & $0.0379(21)$ & $0.00131(12)$ & $1.598(18)$ & $5.4579(80)$ \\
\hline 901.923 & $0.0986(56)$ & $0.490(10)$ & $0.814(12)$ & $0.1036(35)$ & $0.00387(22)$ & $1.506(17)$ & $5.6125(86)$ \\
\hline
\end{tabular}

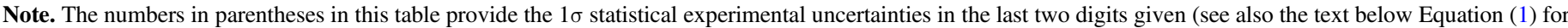
a discussion of the systematic uncertainty of the cross section scale). The systematic uncertainty of the energy scale is $\pm 0.2 \mathrm{eV}$.

(This table is available in its entirety in machine-readable form.)

change of the inner-shell ionization cross section upon excitation of the outermost electrons by only a few eV does not seem very likely.

\subsection{Photoabsorption Cross Section}

Using our calculations, we investigated the effects on our theoretical cross sections due to different populations of the 37 levels of the ground configuration. In each panel of Figure 4, we compare the experimental photoabsorption cross section, shown in blue, with MCDHF and HFR results based on different populations of the fine-structure levels in the ground configuration. These MCDHF results include only photoexcitations into the $3 d, 4 d, 5 d$ shells. The HFR results omit contributions from the $4 d$ and $5 d$ shells but also include photoionization of $M$ and $L$-shell electrons. The increase of the HFR cross section starting around $760 \mathrm{eV}$ is the contribution from the photoionization of $2 p$ electrons. The respective level populations are displayed in the insets of the panels. To account for the uncertainty due to the experimental photon-energy spread and the lifetime broadening, the computed data were convoluted with a Voigt profile, where the full width at half maximum (FWHM) of the Gaussian was chosen as $1.0 \mathrm{eV}$ and a uniform natural line width of $\Gamma=0.4 \mathrm{eV}$ was assumed. These parameters are chosen to match the Gaussian width to the approximate experimental photon spread (see Section 2) and to approximately match the computed lifetimes of the corehole excited configurations (see Section 3) to the Lorentzian width. In addition, the calculated spectra were shifted by $-2.2 \mathrm{eV}$ such that the theoretical and experimental positions of the tallest resonance feature at about $711 \mathrm{eV}$ match.

In the top panel (Figure 4(a)), we assume that only the wellseparated ground level is populated in the initial ion beam. As a consequence, both the MCDHF and HFR calculations overpredict the cross section, especially for the $2 p_{3 / 2}$ excitation at about $711 \mathrm{eV}$. Moreover, the calculated cross sections exhibit more details than the experimental photoabsorption spectrum. Both theories agree very well with each other. However, the $4 d$ and $5 d$ excitations were not included in the HFR calculations and therefore the corresponding resonances are only visible in the MCDHF results. Furthermore, CI between the different $n d$ configurations slightly reduces the MCDHF cross section, as can also be seen in Figure 5. This partially accounts for the lower peak cross section predicted by our MCDHF results as compared with the HFR results seen in Figure 4.

In Figure 4(b) we assume the statistical population of the $3 d^{5}{ }^{6} S_{5 / 2}$ ground level and the $3 d^{5}{ }^{4} G$ first excited multiplet, as seen in the inset. As a consequence, both theories predict that some of the fine structure that is visible in Figure 4(a) cannot be resolved anymore and that the strongest line becomes wider, while its maximum is drastically lowered, in better agreement with the experiment. The same trend continues, when the next two multiplets $\left({ }^{4} P\right.$ and $\left.{ }^{4} D\right)$ are included in the statistical mixture, as seen in Figure 4(c). Compared with the experimental results, the total theoretical cross sections are in good agreement, though too much fine structure still remains visible in the theory. When the statistical average is extended over all 37 fine-structure levels of the ground configuration, the remaining fine structure also vanishes and only six rather broad lines remain, as seen in Figure $4(\mathrm{~d})$. Also noteworthy is that the $2 p_{3 / 2}$ resonance feature is underestimated in this model.

These results show that the assumption of just the ground level being populated is not justified, neither is the assumption of a statistical population of all levels in the ground configuration. Furthermore, a drastic cut in the population, such as in Figures 4(b) and (c) is also a rather unrealistic scenario, especially because only the ground level is energetically well separated. Therefore, a population that gives clear preference to the ground level but also populates all excited levels of the ground configuration seems more appropriate. For this purpose we chose a Boltzmann distribution at a temperature of $30,000 \mathrm{~K}$ with no other justification than the relatively good agreement between the calculated and measured photoabsorption spectra, as seen in Figure 4(e). This temperature also seems plausible in view of the electron energies that have been estimated for plasmas in ECR ion sources (Trassl 2003). At this temperature, the population within any given multiplet is almost statistical, while the population of excited multiplets is suppressed due to their high excitation energies. The result of choosing this distribution and temperature is in good agreement with the experimental results, not only in terms of the maximum value of the cross section but also for the width of the resulting lines. All the following results were computed with this distribution for the population of the 37 fine-structure levels of the ground configuration in the ion beam. 


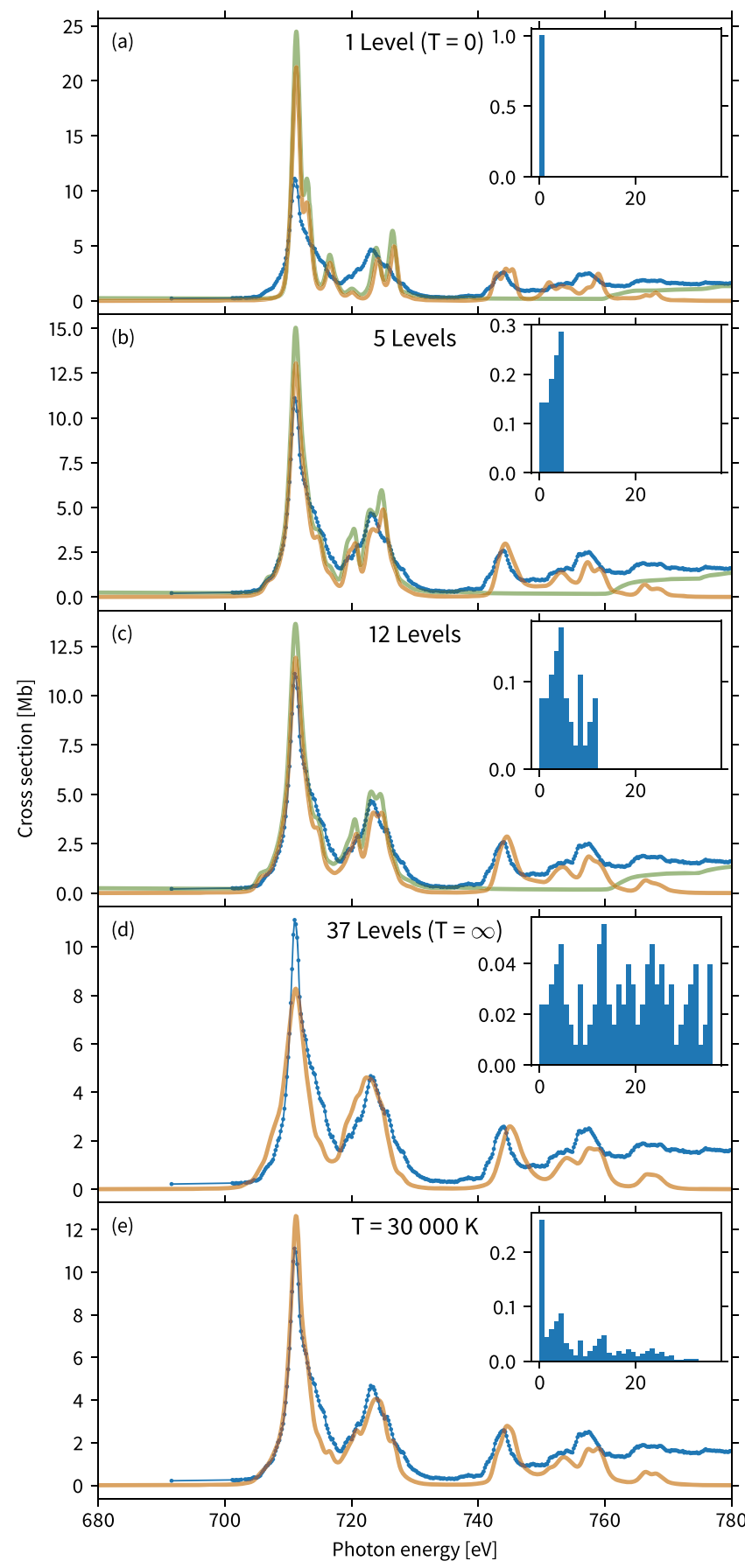

Figure 4. Computed cross section for different populations of the $3 d^{5}$ ground configuration. Panels (a)-(d) are computed with a statistical population of the lowest $N=1,5,12$, and 37 fine-structure levels, respectively, while (e) is based on a Boltzmann distribution at $T=30,000 \mathrm{~K}$. The blue dots are the experimental data from Figure 2, the orange line represents the MCDHF computation of the cross section for photoexcitation and the green curves are the HFR photoabsorption cross sections (photoionization and photoexcitation) not including $4 d$ and $5 d$ excitations. The computed spectra were convoluted with a Voigt profile with a Gaussian FWHM of $1.0 \mathrm{eV}$ and lifetime broadening of $\Gamma=0.4 \mathrm{eV}$. The computed MCDHF and HFR energies have been shifted by $-2.2 \mathrm{eV}$. The inset histograms show the relative population vs. level number for the 37 ground-configuration fine-structure levels that is assumed for each plot.

The positions of the photoexcitation peaks also slightly depend on the population of metastable levels in the ion beam. The strongest shift is observed for the line around $722 \mathrm{eV}$

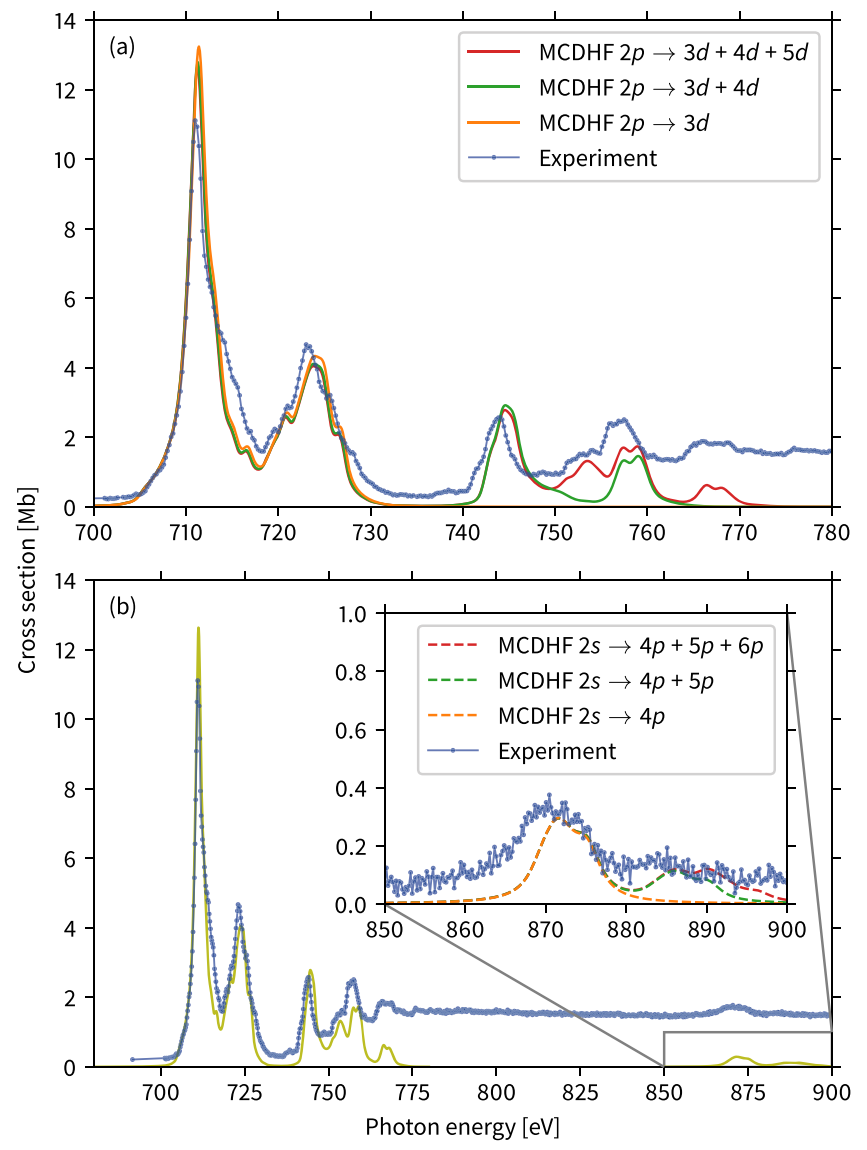

Figure 5. Measured photoabsorption cross section and our MCDHF calculations, including (a) $2 p \rightarrow n d$ resonances and (b) $2 s \rightarrow n p$ resonances. The inset in (b) enlarges the region of the $2 s \rightarrow n p$ resonances. Computed spectra are convoluted with a Voigt profile with a Gaussian FWHM $=1.0 \mathrm{eV}$ and natural line widths of $\Gamma=0.4 \mathrm{eV}$ and $\Gamma=3.5 \mathrm{eV}$ for the $n d$ and $n p$ resonances, respectively. Here, an offset of $-1.4 \mathrm{Mb}$ was added to the experimental data to reduce the $L$ and $M$-shell photoionization background and to facilitate a comparison with the computation. The computed MCDHF energies have been shifted by $-2.2 \mathrm{eV}$. Where the theoretical curves overlap, the orange curve lies on top of the green curve, which lies on top of the red curve.

which is a blend of many transitions. Here, the shift between Figures 4(d) and (e) is about $1.4 \mathrm{eV}$. For the line at approximately $745 \mathrm{eV}$, which primarily arises from $2 p_{3 / 2} \rightarrow 4 d$ excitations, the shift is about $0.5 \mathrm{eV}$. The position of the tallest peak at $711 \mathrm{eV}$, which is associated with $2 p_{3 / 2} \rightarrow 3 d$ excitations, however, is almost constant, shifting by $0.1 \mathrm{eV}$ at most.

Figure 5(a) shows the experimental photoabsorption cross section in the $2 p$-threshold region together with the computed photoexcitation cross section resulting from $2 p \rightarrow n d(n=3$, 4,5 ) excitations. The three lowest lines arise from $2 p \rightarrow 3 d$ or $4 d$ excitations while the higher resonance structures are blends of contributions with different principal quantum numbers of the upper levels.

Figure 5(b) displays the measured and computed cross sections over a larger energy range, that also includes the $2 s$ threshold. The cross sections around the $2 p$ threshold are identical to the ones in Figure 5(a), while the computed data for the $2 s$ core excited levels (inset) are convoluted with a Voigt profile with a Lorentzian width $\Gamma=3.5 \mathrm{eV}$ to account for the much faster decay of those states (see Section 3.1). Again, the 


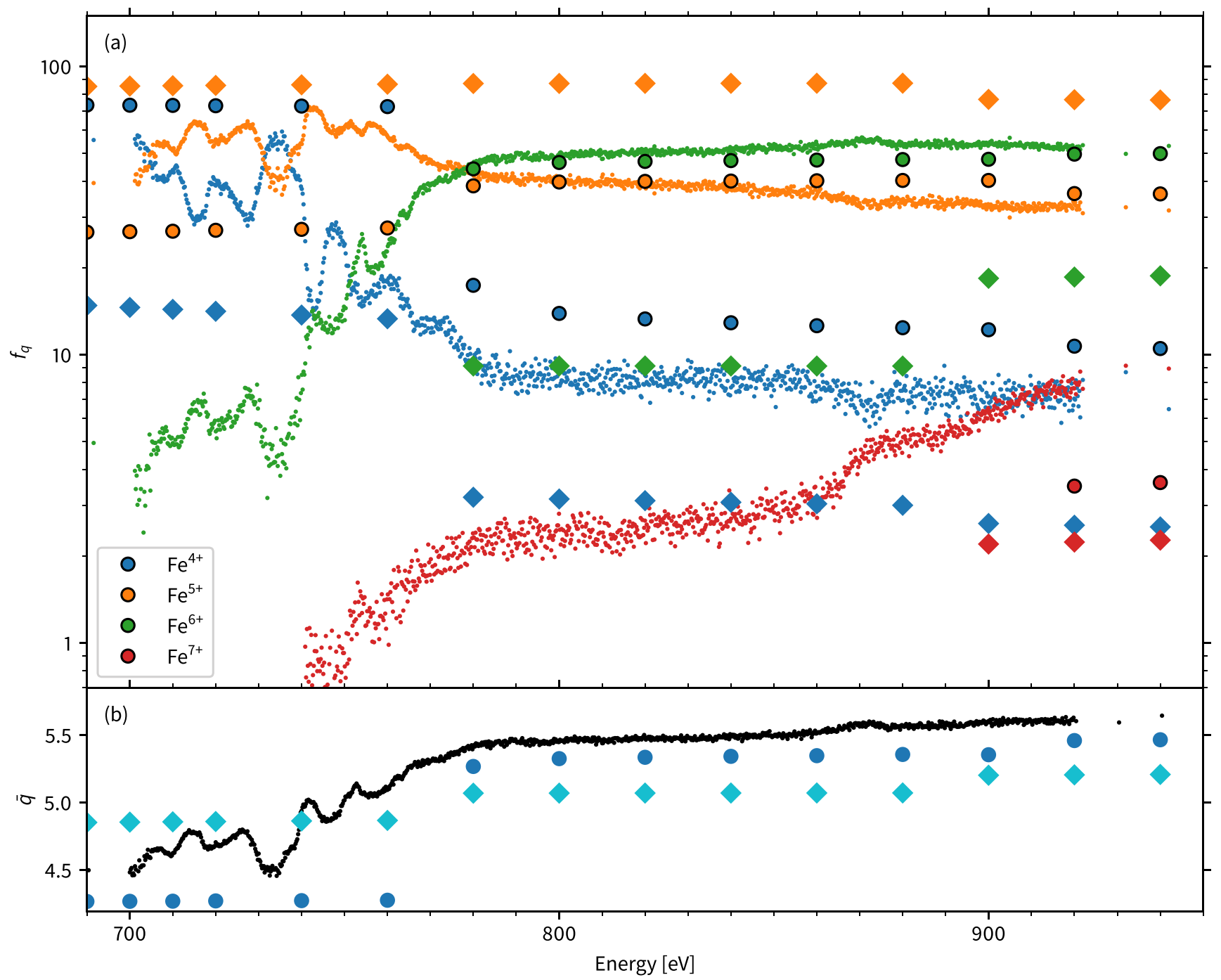

Figure 6. (a) Product charge-state fractions, $f_{q}$, in percentage for the four charge states $q=4,5,6$, and 7. Experimental results (small circles) are compared with our computations (large circles) for direct ionization of a single electron using the shake-down Auger model and to the results by Kaastra \& Mewe (1993) weighted by the relative direct photoionization cross sections of Verner et al. (1993) (diamonds). (b) Mean charge state from the experimental data (small black circles) and our cascade calculations (large blue circles). The diamonds are again the results from Kaastra \& Mewe (1993) combined with the cross sections of Verner et al. (1993).

lowest three $n p(n=4,5,6)$ shells were taken into account. As seen from the inset of this figure, these contributions are also visible in the experimental data.

\subsection{Product Charge State Fractions}

The product charge-state fractions, i.e., the probabilities of an atom to decay into charge state $q$, can be derived as $f_{q}\left(E_{\mathrm{ph}}\right)=\sigma_{q} / \sigma_{\Sigma}$. Here, $\sigma_{q-3}=\sigma_{m}$ are the measured partial cross sections and $E_{\mathrm{ph}}$ signifies the photon energy. The key feature of the $f_{q}$ values is that the systematic uncertainty of the absolute cross-section scale cancels out. Furthermore, the $f_{q}$ fractions can be used to calculate the mean product charge state as

$$
\bar{q}\left(E_{\mathrm{ph}}\right)=\sum_{q=4}^{8} q f_{q}=\frac{1}{\sigma_{\Sigma}} \sum_{m=1}^{5}(m+3) \sigma_{m} .
$$

Figure 6(a) shows the product charge-state fractions for the overall ionization process and Figure 6(b) the mean charge state $\bar{q}$ (see also Table 1). In addition to the experimental data, which are displayed by small circles, both figures compare our computed results for these quantities (large circles) with the results obtained as a combination of the theoretical cross sections for photoionization by Verner et al. (1993) and the cascade calculations by Kaastra \& Mewe (1993) (diamonds).

Here we compute the theoretical product charge-state fractions due either to photoionization or photoexcitation. Because of the above mentioned issues in the computation of absolute photoionization cross sections, we did not add together the contributions from photoionization and photoexcitation.

When considering only photoionization, we calculate the product charge-state fractions using

$$
f_{q}\left(E_{\mathrm{ph}}\right)=\frac{1}{\sigma_{\mathrm{tot}}\left(E_{\mathrm{ph}}\right)} \sum_{k} \sigma_{k}\left(E_{\mathrm{ph}}\right) F_{k, q}
$$

where $\sigma_{k}\left(E_{\mathrm{ph}}\right)$ is the cross section for direct photoionization of an electron from subshell $k$ versus photon energy, and the total photoionization cross section is again obtained by summing over all subshells $\sigma_{\mathrm{tot}}\left(E_{\mathrm{ph}}\right)=\sum_{k} \sigma_{k}\left(E_{\mathrm{ph}}\right) . F_{k, q}$ denotes the fraction $\mathrm{Fe}^{q+}$ produced after the removal of an electron from subshell $k$ of $\mathrm{Fe}^{3+}$ and is discussed in the next subsection. 
Table 2

Comparison of the Theoretical Photoionization Branching Ratios $\sigma_{k} / \sigma_{\text {tot }}$ from this Work with the Results of Verner et al. (1993)

\begin{tabular}{lccccc}
\hline \hline Energy $(\mathrm{eV})$ & $2 s$ & $2 p$ & $3 s$ & $3 p$ & $3 d$ \\
\hline \multicolumn{5}{c}{ This work } \\
\hline 690 & 0 & 0 & 20 & 68 & 13 \\
840 & 0 & 82 & 4 & 12 & 1.8 \\
960 & 12 & 74 & 3.5 & 10 & 1.2 \\
\hline \multicolumn{7}{c}{ Verner et al. (1993) } \\
\hline 690 & 0 & 0 & 20 & 66 & 15 \\
840 & 0 & 87 & 2.7 & 8.4 & 1.5 \\
960 & 13 & 76 & 2.5 & 7.4 & 1.1 \\
\hline
\end{tabular}

Note. The results are given in percentage.

The quantities $\sigma_{k}\left(E_{\mathrm{ph}}\right) / \sigma_{\mathrm{tot}}\left(E_{\mathrm{ph}}\right)$ represent the photoionization branching ratios. We utilized the PHOTO component of the RATIP code (Fritzsche 2012) to compute these quantities from our MCDHF wave functions for all subshells for which ionization is possible in the given energy range. In the upper part of Table 2, we show these results for three energies that are representative for the three main regions covered in the experiment: below the $2 p$ threshold, between the $2 p$ and $2 s$ threshold, and above the latter. At these energies, photoionization dominates over photoexcitation. The lower part of Table 2 shows the theoretical results obtained by Verner et al. (1993), using a relativistic Hartree-Dirac-Slater method. Generally, their findings agree well with our results. The rather small differences could be due to differences in the treatment of relaxation effects.

When considering only photoexcitation, we replace $\sigma_{k}\left(E_{\mathrm{ph}}\right) /$ $\sigma_{\text {tot }}\left(E_{\mathrm{ph}}\right)$ in Equation (3) with the theoretical fractional populations from the photoexcitation transition rates. The definition of $F_{k, q}$ remains unchanged.

\subsection{Cascade Models}

The branching fractions $F_{k, q}$ were computed for all innershell holes that can be created at the photon energies under consideration by utilizing the MCDHF cascade calculations explained in Section 3.1. Previous cascade calculations were performed by Kaastra \& Mewe (1993) to predict the branching fractions after inner-shell ionization for various transition metal elements. Their results for $\mathrm{Fe}^{3+}$ are shown in the lowest part of Table 3.

In our most straightforward Auger model, we built the cascade tree by including all energetically allowed two-electron Auger processes. The results from this model, denoted as twoelectron Auger, are shown in the middle part of Table 3. They agree to a large extent with the earlier results of Kaastra \& Mewe (1993). One notable exception concerns the decay of $3 p$ holes. According to our computations, the corresponding highlying levels are above the ionization threshold (see Figure 3), but they do not get populated to a significant extent in the photoionization process, so that almost all $3 p$ holes formed produce only $\mathrm{Fe}^{4+}$. In contrast, Kaastra \& Mewe (1993) find that a $3 p$ hole will autoionize and, thus, lead to the formation of $\mathrm{Fe}^{5+}$.

For the higher product charge states, there are several innershell hole configurations that, for energetic reasons, are partially forbidden to decay via two-electron Auger processes.
Table 3

Computed Branching Fractions $F_{k, q}$, given here in Percentage, of an Inner Shell Hole Created in Subshell $k$ by Direct (Single) Photoionization

\begin{tabular}{|c|c|c|c|c|c|}
\hline$k / q$ & $\mathrm{Fe}^{4+}$ & $\mathrm{Fe}^{5+}$ & $\mathrm{Fe}^{6+}$ & $\mathrm{Fe}^{7+}$ & $\mathrm{Fe}^{8+}$ \\
\hline \multicolumn{6}{|c|}{ This work (shake-down) } \\
\hline $2 s$ & $\cdots$ & 2.5 & 64 & 33.6 & $\cdots$ \\
\hline $2 p$ & $\cdots$ & 47 & 53 & $\cdots$ & $\cdots$ \\
\hline $3 s$ & $\cdots$ & 100 & $\cdots$ & $\cdots$ & $\cdots$ \\
\hline $3 p$ & 100 & $\cdots$ & $\cdots$ & $\cdots$ & $\cdots$ \\
\hline \multicolumn{6}{|c|}{ This work (two-electron Auger) } \\
\hline $2 s$ & $\cdots$ & 4.0 & 95 & 1.1 & $\cdots$ \\
\hline $2 p$ & $\cdots$ & 89 & 11 & $\cdots$ & $\cdots$ \\
\hline $3 s$ & $\cdots$ & 100 & $\cdots$ & $\cdots$ & $\cdots$ \\
\hline $3 p$ & 100 & $\cdots$ & $\cdots$ & $\cdots$ & $\cdots$ \\
\hline \multicolumn{6}{|c|}{ Kaastra \& Mewe (1993) } \\
\hline $2 s$ & $\cdots$ & 0.3 & 83.0 & 14.3 & 0.04 \\
\hline $2 p_{1 / 2}$ & 1.8 & 87.2 & 10.5 & 0.54 & $\cdots$ \\
\hline $2 p_{3 / 2}$ & 1.1 & 84.9 & 13.3 & 0.67 & $\ldots$ \\
\hline $3 s$ & $\ldots$ & 100 & $\ldots$ & $\ldots$ & $\cdots$ \\
\hline $3 p$ & $\ldots$ & 100 & $\ldots$ & $\ldots$ & $\cdots$ \\
\hline
\end{tabular}

Figure 3 displays three examples that are marked in red and that arise in the decay of the $2 s^{2} 2 p^{5} 3 s^{2} 3 p^{6} 3 d^{6}$ configuration (configuration 2 in Figure 3). For example, the higher-lying fine-structure levels of the $2 s^{2} 2 p^{6} 3 s^{2} 3 p^{4} 3 d^{6}$ configuration (configuration 7 in Figure 3) can decay via a two-electron Auger process to $2 s^{2} 2 p^{6} 3 s^{2} 3 p^{5} 3 d^{4}$ (configuration 13 in Figure 3), while this decay path is forbidden for the lower lying levels. However, these lower levels are still above the ionization threshold for $\mathrm{Fe}^{4+}$ forming $\mathrm{Fe}^{5+}$. Therefore, they can decay by a three-electron Auger process where a third electron undergoes a shake-down $3 d \rightarrow 3 p$ transition filling the $3 p^{4}$ double vacancy and thereby forming the ground configuration of $\mathrm{Fe}^{5+}$ (configuration 12 in Figure 3). In general, such threeelectron Auger processes are expected to be slow compared with a two-electron Auger process. Nevertheless, they can still be faster than the competing radiative processes that would result in $\mathrm{Fe}^{4+}$ product ions. The precise computation of the Auger transition rates including a shake-down transition is rather challenging due to complex correlation patterns (Andersson et al. 2015; Schippers et al. 2016a; Beerwerth \& Fritzsche 2017). Here we assume that the radiative losses are still negligible, so that all levels that are energetically allowed to autoionize will do so. In the following we will refer to this extended cascade decay tree as "shake-down." The resulting branching fractions $F_{k, q}$ are shown in the upper part of Table 3. They give rise to drastic changes in the ion yield from $2 p$ and $2 s$ holes. For example, the yields of $\mathrm{Fe}^{6+}$ and $\mathrm{Fe}^{7+}$, respectively, are significantly increased.

We can combine the fractions $F_{k, q}$ with the computed photoionization branching ratios $\sigma_{k} / \sigma_{\text {tot }}$ from Table 2 to model the full decay tree and compare the resulting ion yields and mean charge state versus photon energy to the experimental results. The resulting product charge-state fractions are given in Table 4 for both photoexcitation of the initial ion as well as for direct photoionization. For both cases, the results are again given for the two cascade models introduced before, with and without shake-down transitions included. In the case of direct ionization, the results are given for three energies, below the $2 p$ threshold, between the $2 p$ and $2 s$ thresholds, and above the latter. As already expected from the ion fractions $F_{k, q}$ in 
Table 4

Experimental and Theoretical Product Charge-state Fractions $f_{q}$ upon Photoexcitation or Direct Photoionization of $\mathrm{Fe}^{3+}$ by a Photon of the given Energy

\begin{tabular}{|c|c|c|c|c|c|}
\hline Energy $(\mathrm{eV})$ & $\mathrm{Fe}^{4+}$ & $\mathrm{Fe}^{5+}$ & $\mathrm{Fe}^{6+}$ & $\mathrm{Fe}^{7+}$ & $\mathrm{Fe}^{8+}$ \\
\hline \multicolumn{6}{|c|}{$2 p \rightarrow 3 d$ Resonances (experiment) } \\
\hline 711 & 45. & 50. & 5. & 0.3 & 0.01 \\
\hline 723 & 34. & 59. & 7. & 0.3 & 0.01 \\
\hline \multicolumn{6}{|c|}{$2 p \rightarrow 3 d$ Resonances (shake-down) } \\
\hline 711 & 41.9 & 56.7 & 1.3 & $\cdots$ & \\
\hline 723 & 40.3 & 56.9 & 2.7 & $\cdots$ & \\
\hline \multicolumn{6}{|c|}{$2 p \rightarrow 3 d$ Resonances (two-electron Auger) } \\
\hline 711 & 66.9 & 31.7 & 1.3 & $\cdots$ & \\
\hline 723 & 54.6 & 42.7 & 2.8 & $\cdots$ & \\
\hline \multicolumn{6}{|c|}{ Direct Ionization (experiment) } \\
\hline 690 & 55.6 & 39.4 & 4.9 & 0.2 & 0.001 \\
\hline 840 & 7.7 & 38.2 & 51.4 & 2.7 & 0.1 \\
\hline 960 & 6.9 & 32.2 & 51.5 & 9.4 & 0.4 \\
\hline \multicolumn{6}{|c|}{ Direct Ionization (shake-down) } \\
\hline 690 & 73.4 & 26.6 & 0.0 & 0.0 & \\
\hline 840 & 12.9 & 40.0 & 47.1 & 0.0 & \\
\hline 960 & 10.4 & 35.9 & 49.9 & 3.8 & \\
\hline \multicolumn{6}{|c|}{ Direct Ionization (two-electron Auger) } \\
\hline 690 & 73.4 & 26.6 & 0.0 & 0.0 & \\
\hline 840 & 12.9 & 76.9 & 10.1 & 0.0 & \\
\hline 960 & 10.4 & 69.2 & 20.2 & 0.2 & \\
\hline
\end{tabular}

Note. The results are given in percentage.

Table 3, the total product charge-state fractions from the two models differ dramatically.

The theoretical product charge-state fractions due to photoionization only are graphically presented in Figure 6, together with the experimental data. The small circles are the experimental data, while the large circles are our theoretical values using the shake-down Auger model. Our theoretical data do not reproduce the measured resonance structures because we account only for photoionization here and do not include the effects of photoexcitation. The diamonds are the theoretical results that are obtained by combining the photoionization branchings from Verner et al. (1993) with the cascade calculations by Kaastra \& Mewe (1993). For this last case, the resulting charge-state fractions disagree significantly with the experiment. This was also seen for the respective calculations for $\mathrm{Fe}^{+}$by Schippers et al. (2017). The mean charge state from the combined Verner et al. (1993) and Kaastra \& Mewe (1993) results is significantly overestimated below the $2 p$ ionization threshold and the step at the ionization threshold is much less pronounced than in the experimental data. Above the $2 p$ ionization threshold, the mean charge state is significantly underestimated. This behavior arises because the calculations by Kaastra \& Mewe (1993) predict the fraction of $\mathrm{Fe}^{5+}$ to be about a factor of two too high, while the predicted fraction of $\mathrm{Fe}^{6+}$ is about an order of magnitude too low. Similarly, both the predicted $\mathrm{Fe}^{4+}$ and $\mathrm{Fe}^{7+}$ charge-state fractions are also too low. The low $\mathrm{Fe}^{4+}$ fraction is a consequence of the autoionizing behavior of $3 p$ holes that was predicted by Kaastra \& Mewe (1993) and that disagrees with our present findings.
Figure 6 shows that our calculations represent a significant improvement over the previous computations by Kaastra \& Mewe (1993). Most notably, as can be seen in Figure 6(b), the pronounced step in the mean charge state at the $2 p$ ionization threshold is clearly reproduced and is hence in much better agreement with experiment, but still somewhat underestimated. As can be seen in Figure 6(a), our calculations also predict the charge-state fractions more accurately than the previous theory. Most importantly, the two strongest channels, $\mathrm{Fe}^{6+}$ and $\mathrm{Fe}^{5+}$, are predicted quite well and in the correct order. However, the production of $\mathrm{Fe}^{4+}$ is still slightly overestimated, and the production of the highest measured charge states $(q=7,8)$ is significantly underestimated. The main reason that our computations are in better agreement with the experiment than previous theory is the incorporation of shake-down transitions and of more precise transition energies and rates from our fine-structure resolved treatment.

\section{Summary and Conclusions}

We have measured relative cross sections for up to five-fold ionization of $\mathrm{Fe}^{3+}$ ions after resonant $L$-shell photoexcitation or direct photoionization. We have used a photon-ion mergedbeams technique. The present measurements are a continuation of the earlier work on $\mathrm{Fe}^{+}$(Schippers et al. 2017). We observed strong ionization resonances due to $2 p \rightarrow n d$ excitations, where contributions by $n=3,4$, and 5 could be identified with the help of MCDHF calculations. Around the $2 s$ ionization threshold, we were able to identify $2 s \rightarrow n p$ resonances, where the $4 p$ contribution can be clearly seen and higher shells contribute to some weak and broad feature.

Furthermore, we performed extensive calculations of the deexcitation cascades that follow upon the creation of holes in the $2 s$ and $2 p$ shells. Our computed product charge-state fractions agree well with the experimental results, where we found that the contribution of several three-electron Auger processes is the likely main reason why earlier theory based on cascade branching fractions by Kaastra \& Mewe (1993) and photoionization cross sections by Verner et al. (1993) fail to reproduce the current experimental results. Despite these improvements, our current Auger models show notable deficiencies in describing the formation of the highest charge states, in this case $\mathrm{Fe}^{7+}$ and $\mathrm{Fe}^{8+}$. In our models $\mathrm{Fe}^{8+}$ is not included due to energy conservation, and important decay paths leading to $\mathrm{Fe}^{7+}$ are still missing. The starting point for including these charge states into an Auger model would be to include shake-up transitions in the Auger decay of $2 p$ vacancies or direct double-Auger decay processes of $2 p$ vacancies.

The computation of the photoabsorption spectra is complicated by the presence of ions in metastable levels in the experiment. From the comparison with experiment, this effect is found to be more severe than in the previous study on $\mathrm{Fe}^{+}$. Still even with these experimental issues, computations of resonant photoabsorption spectra agree reasonably well with experiment when all 37 fine-structure levels of the ground configuration are assumed to be populated at a temperature of $30,000 \mathrm{~K}$ in the ion beam. Additionally, because the $\mathrm{Fe}^{3+}$ resonance positions are significantly different from the $\mathrm{Fe}^{+}$resonance positions published before (Schippers et al. 2017), it should still be possible to identify individual signatures from both charge states in X-ray photoabsorption or emission spectra. We will discuss this aspect in more depth in a future publication where we will 
also present experimental and theoretical data for single and multiple ionization of $\mathrm{Fe}^{2+}$. Lastly, our benchmarked theoretical results are also being incorporated into models for X-ray absorption in the ISM (T. Kallman 2019, private communication) and are available upon request.

This research was carried out at the light source PETRA III at DESY, a member of the Helmholtz Association (HGF). We would like to thank G. Hartmann, F. Scholz, and J. Seltmann, for assistance in using beam line P04. This research has been funded in part by the German Federal Ministry for Education and Research (BMBF) within the "Verbundforschung" funding scheme under contracts 05K16GUC, 05K16RG1, and 05K16SJA. A.M. acknowledges support by Deutsche Forschungsgemeinschaft. S.B. and K.S. would like to thank SFB 755, "Nanoscale photonic imaging, project B03" for financial support and acknowledge funding from the Initiative and Networking Fund of the Helmholtz Association. D.W.S. was supported, in part, by the NASA Astrophysics Research and Analysis program and the Astrophysics Data Analysis Program.

\section{ORCID iDs}

Randolf Beerwerth (1) https://orcid.org/0000-0001-5100-4229

Ticia Buhr (1) https://orcid.org/0000-0003-3337-1740

Alexander Perry-Sassmannshausen (10 https://orcid.org/00000002-0700-3875

Sebastian O. Stock (D) https://orcid.org/0000-0001-9629-9630

Sadia Bari (iD https://orcid.org/0000-0003-3985-2051

Kristof Holste (i) https://orcid.org/0000-0001-8809-1696

A. L. David Kilcoyne (i) https://orcid.org/0000-0002-

8805-8690

Daniel Wolf Savin (10 https://orcid.org/0000-0002-1111-6610

Michael Martins (ib https://orcid.org/0000-0002-1228-5029

Alfred Müller (iD https://orcid.org/0000-0002-0030-6929

Stephan Fritzsche (i) https://orcid.org/0000-0003-3101-2824

Stefan Schippers (i) https://orcid.org/0000-0002-6166-7138

\section{References}

Andersson, J., Beerwerth, R., Linusson, P., et al. 2015, PhRvA, 92, 023414 Beerwerth, R., \& Fritzsche, S. 2017, EPJD, 71, 253

Behar, E., Sako, M., \& Kahn, S. M. 2001, ApJ, 563, 497

Blancard, C., Cubaynes, D., Guilbaud, S., \& Bizau, J.-M. 2018, ApJ, 853, 32

Buth, C., Beerwerth, R., Obaid, R., et al. 2018, JPhB, 51, 055602

Cowan, R. D. 1981, The Theory of Atomic Structure and Spectra (Berkeley, CA: California Univ. Press) atomic-structure code accessible via http:// aphysics2.lanl.gov/tempweb/lanl/

Fritzsche, S. 2001, JESRP, 116, 1155

Fritzsche, S. 2012, CPC, 183, 1525

Froese Fischer, C., Rubin, R. H., \& Rodriguez, M. 2008, MNRAS, 391, 1828

Grant, I. P. 2007, Relativistic Quantum Theory of Atoms and Molecules: Theory and Computation, Vol. 40 (New York: Springer Science \& Business Media)

Hirsch, K., Zamudio-Bayer, V., Ameseder, F., et al. 2012, PhRvA, 85, 062501

Holczer, T., Behar, E., \& Kaspi, S. 2005, ApJ, 632, 788

Jönsson, P., Gaigalas, G., Bieroń, J., Froese Fischer, C., \& Grant, I. P. 2013, CPC, 184, 2197

Jönsson, P., He, X., Fischer, C. F., \& Grant, I. 2007, CoPhC, 177, 597

Kaastra, J. S., \& Mewe, R. 1993, A\&AS, 97, 443

Kramida, A., Ralchenko, Y., Reader, J. \& NIST ASD Team 2018, NIST Atomic Spectra Database (version 5.5.2), Tech. Rep., National Institute of Standards and Technology, http://physics.nist.gov/asd

Lee, J. C., Xiang, J., Ravel, B., Kortright, J., \& Flanagan, K. 2009, ApJ, 702, 970

McGuire, E. J. 1972, PhRvA, 5, 1043

Miedema, P. S., \& de Groot, F. M. 2013, JESRP, 187, 32

Müller, A., Bernhardt, D., Borovik, A., Jr., et al. 2017, ApJ, 836, 166

Nahar, S. N. 2006, A\&A, 448, 779

Reilman, R. F., \& Manson, S. T. 1979, ApJS, 40, 815

Schippers, S., Beerwerth, R., Abrok, L., et al. 2016a, PhRvA, 94, 041401

Schippers, S., Kilcoyne, A. L. D., Phaneuf, R. A., \& Müller, A. 2016b, ConPh, 57,215

Schippers, S., Martins, M., Beerwerth, R., et al. 2017, ApJ, 849, 5

Schippers, S., Ricz, S., Buhr, T., et al. 2014, JPhB, 47, 115602

Simon, M. C., Crespo López-Urrutia, J. R., Beilmann, C., et al. 2010, PhRvL, 105,183001

Trassl, R. 2003, in ECR Ion Sources, ed. F. J. Currell (Dordrecht: Springer Netherlands), 3

Verner, D. A., \& Yakovlev, D. G. 1995, A\&AS, 109, 125

Verner, D. A., Yakovlev, D. G., Band, I. M., \& Trzhaskovskaya, M. B. 1993, ADNDT, 55, 233

Viefhaus, J., Scholz, F., Deinert, S., et al. 2013, NIMA, 710, 151 